\title{
EDITORIAL
}

\section{IN THE JUNE 2007 ISSUE OF CLINICS}

\author{
Mauricio Rocha-e-Silva, Editor
}

In this issue of CLINICS, the cover illustrates data from a basic science piece of research conducted by Higa et al who determined the protective effects of ascorbic acid on intestinal morphology during ischemia-reperfusion injury in rats. Villous architecture, crypts, necrosis, hemorrhagic infarcts and inflammatory cells at the mesenteric and antimesenteric borders of the small intestine were quantitatively evaluated. Ascorbic acid caused (i) a significant reduction of antimesenteric villous hemorrhagic infarction after ischemia-reperfusion, and (ii) reduction of villous necrosis at both borders. A quantitative evaluation of parameters reinforces these conclusions. They conclude that ascorbic acid pretreatment has a protective effect against the intestinal morphological lesions induced by ischemia-reperfusion injury in rats.

In this issue CLINICS publishes a total of eighteen original research papers, thirteen of which report data on Clinical Sciences.

Sanches et al evaluated the liquid chromatography analytical micromethod for quantification of propranolol in children submitted to corrective surgery for tetralogy of Fallot and found that it yields high selectivity, recovery, sensitivity, linearity, precision and accuracy. They conclude that monitoring of plasma concentrations of propranolol in these patients showed that after the last preoperative dose concentration declined from the beginning of surgery to the second postoperative day, suggesting that, once redistribution was restored, propranolol washout was complete.

Lima et al evaluated prevalence of allergic disease among 6 and 7 year-old students from the western districts of São Paulo City and compared these data with those obtained in the International Study of Asthma and Allergies in Childhood (ISAAC) phase I, performed in the central-southern districts of São Paulo. Out of 5,040 distributed questionnaires 3,312 were returned. The comparison showed higher asthma prevalence and severity, but lower values for rhinitis and eczema in this more recent study. The male gender predominated in all positive responses regarding asthma and rhinitis.

Coghi et al measured quality of life in 75 Brazilian ambulatory adults with atopic dermatitis using a Quality of Life Generic Questionnaire and a 10-item Dermatology Life Quality Index Questionnaire. Quality of life and disease control were found to be related with low scores to both instruments, but correlation was better with the former. They conclude that quality of life is affected in adult atopic patients, with respect to disease severity and to mental components, but with varying effects in patient subgroups. Some components appeared to mask the exact relationship between quality of life and disease severity.

Anacleto et al determined the occurrence of the dispensing error rate and endeavored to identify factors associated with them

Hospital das Clínicas, São Paulo University Medical School - São Paulo/ SP, Brazil.

Email: mrsilva36@hcnet.usp.br through a cross-sectional study performed on 422 prescription order forms at a general hospital in Belo Horizonte that uses a mixed system of collective and individualized dosing. The study registered $81.8 \%$ of prescriptions with at least 1 dispensing error. Errors were more frequent in the pre-typed prescription order forms, in those with 9 or more drugs, and in those for injectable drugs. One of the teams of professionals exhibited a higher chance of errors. They conclude that the dispensing system can produce many latent failures and does not have an adequate control; several conditions predispose to the occurrence of errors, contributing to the high rate reported.

Colombo Jr et al retrospectively analyzed the data of 88 patients undergoing radical nephrectomy for renal cell carcinoma prior to January 2000. Of these, 45 patients underwent laparoscopic radical nephrectomy, and 43 patients underwent open radical nephrectomy. The 5-year oncological outcomes of these patients show that laparoscopic and open radical nephrectomy confer equivalent 5-year oncological outcomes. Laparoscopic surgery was quicker, resulted in less blood loss, shorter hospital stay, and reduced costs.

Nascimento et al investigated the plasma levels and the pharmacokinetics of cefuroxime during and after surgery in adult patients with elective indication for coronary artery bypass grafting. They find that kinetic of disposition of cefuroxime remains unaltered in these patients. In order to reduce the fluctuation in plasma concentrations so as to ensure proper antibiotic prophylaxis in the peri-operative period, the dose regimen should be reviewed.

Rabelo and Schochat developed a Brazilian Portuguese program of compressed speech test which they tested in 144 normal-hearing adult volunteers in order to verify which of the compressed lists $(50 \%, 60 \%$, or $70 \%)$ is the most appropriate to be part of a set of auditory processing tests.

Monosyllables and disyllables were used in 8 previously established sequences, and the results were compared with respect to the initial ear, to the order of presentation, and to the kind of test. No significant differences were found between ears. The $50 \%, 60 \%$ and $70 \%$ presentation order produced a better average of correct responses than the $70 \%, 60 \%$ and $50 \%$ one. Significant differences were observed between the results of the tests comprising lists of monosyllables compared to those comprising disyllables. They conclude that monosyllabic and disyllabic lists with $60 \%$ compression are more stable than others, with an average of correct responses around $90 \%$, and should form the base of a set of auditory tests.

Preti et al evaluated knowledge concerning diabetic retinopathy among the physicians present at a on Diabetes Congress though a questionnaire administered to 168 endocrinologists about their experience and management of patients with diabetes mellitus. They found that $36.9 \%$ correctly referred patients with diabetes type 1 to an ophthalmologist, whereas $86.9 \%$ referred patients with the type 2 disorder as recommended by the American Academy of Ophthalmology. More physicians who had 
received their degree less than 5 years previously implemented the correct practice as opposed to those who had received their degree 20 or more years ago They conclude that medical knowledge among medical practitioners and endocrinologists on preventive measures and periodicity of diabetic retinopathy examinations is far from ideal for diabetes type 1 , but satisfactory for diabetes type 2 and suggest that refresher courses emphasizing the correct management of diabetic patients are necessary.

Paschoal et al describe the initial steps of the construction process of a quality of life evaluation instrument for the elderly, the construction methodology, and the generation of relevant items. The first step was to conceptualize and define the construct, determining how much the elderly are able to perform of what they believe to be important in their lives and whether they are satisfied with what was possible to perform. The next step was to select and describe the construction methodology. From 1032 answers by older people, 138 relevant items for the construct were identified by the process of item generation. The next step will consist of administering the resulting list to a sample of elderly people for item reduction and distribution of items into dimensions.

Fonseca et al retrospectively evaluated the epidemiology and the mechanisms involved in the occurrence of facial fractures among car occupants in the metropolitan area of São Paulo. Data were collected from 56 patients admitted with facial fractures to the emergency room of Hospital das Clínicas who had been inside cars involved in accidents. Grouped according to seating position at the time of the accident and the wearing of seat belts, data showed that the 323 fracture lines which occurred among the 56 patients distributed with higher risk in the group of rearseat passengers who were not wearing seat belts, followed by the group of drivers not wearing seat belts, the group of front-seat passengers not wearing seat belts, the group of drivers wearing seat belts and, finally, the group of front-seat passengers wearing seat belts. They suggest that an educational campaign should be envisaged and that the use of seat belts in the back seat should be strongly encouraged.

Marie et al examined the risk for ischemic stroke associated to a common polymorphism in the gene encoding 5,10methylenetetrahydrofolate reductase $\mathrm{C} 677 \mathrm{~T}$, which predisposes carriers to hyperhomocysteinemia. The study was performed in 127 subjects presenting ischemic stroke and in 126 normal control volunteers and found that the homozygous 5,10methylenetetrahydrofolate reductase C677T genotype was not a risk factor for ischemic stroke in these Brazilian subjects.

Carvalho and Kara José assessed the need for emergency care in a tertiary hospital by analyzing files from the Ophthalmology Emergency Room of the University of the São Paulo Medical School General Hospital, through a cross-sectional analytic study of the treatment complexity level carried out in a readily available sample of 574, of patients seen at the Ophthalmology Emergency Room during a typical week. Of the 574 treated patients, they note that most could have been diagnosed and treated in primary or secondary care units, which demonstrates the lack of referral services and the poor structure of the Brazilian Public Healthcare System, which overloads the tertiary care facilities, where costs for human resources, materials, and other items are higher.

Bricks et al evaluated the reliability of information provided by 204 parents about the history of varicella in their previously healthy children attending two municipal day-care centers of São Paulo. A standardized form was filled out with information regarding age, sex, history of varicella and other diseases, drug use and antecedent of immunization. Subsequently children were submitted to medical evaluation, physical examination, checking of immunization records, and to the collection of $5 \mathrm{ml}$ of blood for ELISA (in house) varicella test. The predictive positive and negative values of the information were $90 \%$ and $93 \%$, respectively which led to the conclusion that information about history of varicella informed by parents of children attending day care centers was highly reliable and useful to establish recommendations on varicella blocking immunization in day-care centers.

In addition to the highlighted article CLINICS publishes four more Basic Science contributions.

Nakagawa et al examined the effects of cecal ligation and puncture and subsequent necrotic tissue resection and peritoneal lavage on specific laboratory parameters and on leukocyte-endothelial interactions in the rat mesentery. Cecal ligation and puncture per se increased the number of rolling, adherent, and migrating leukocytes, elevated blood glucose, lactate, and white cell count; it also caused functional alterations, characterized by impaired alertness and mobility, and presence of piloerection, diarrhea, encrusted eyes, and dirty nose and tail. Necrotic tissue resection and peritoneal lavage normalized the number of rolling, adherent, and migrated leukocytes in the mesentery; glycemia; lactate; and white blood cell count were also back to control. Functional alterations were likewise reversed. They conclude that the local and systemic inflammation induced by cecal ligation and puncture is overcome by necrotic tissue resection and peritoneal lavage.

Barros et al tested the possibility of obtaining a practical and stable model of hyperinsulinemia and hyperglycemia in hamsters, substituting the drinking water by $10 \%$ or $20 \%$ fructose solutions for a period of 2, 4, or 6 months. Compared to controls, animals that drank $10 \%$ or $20 \%$ fructose solution instead of water had significantly greater weight gain, and fasting plasma glucose. An analysis of the effects of reperfusion, after $30 \mathrm{~min}$ of ischemia, resulted in an immediate but reversible increase in postcapillary leakage which was larger in the fructose treated animals. They suggest that this procedure, namely, chronic administration fructose may be useful to experimentally induce a stable hamster model of hyperinsulinemia and hyperglycemia, similar to human type 2 diabetes mellitus. They claim to have generated a model which may facilitate the study of basic mechanisms of hyperglycemia and hyperinsulinemia affecting the microvasculature.

Mota e Albuquerque et al tested an intra-articular reconstruction of the anterior cruciate ligament of the knee in $10 \mathrm{hu}-$ man cadavers by replacing 2 anterior cruciate ligament bundles, with the purpose of producing a surrogate that would be structurally more similar to the anatomy of the anterior cruciate ligament and would provide the knee with more stability. They conclude that results obtained did not show superiority of doublebundle reconstruction over single-bundle reconstruction, and that neither technique provided the knee with the same stability and rigidity afforded by the intact anterior cruciate ligament.

Pazetti et al assayed the effects of cyclosporin A on mucus secretion from goblet cells and on mucociliary transport in situ in a murine model, and found that cyclosporine blood concentration throughout the treatment was therapeutically adequate. They observed that the transport rate of mucus was significantly lower in the cyclosporin A treated group. Mucus quantity measurements showed a significant decrease of both acid and neutral mucus production from goblet cells in the animals submitted to cyclosporin A therapy. They conclude that cyclosporin A plays an important role in the impairment of the mucociliary clearance in rats by reducing both acid and neutral mucus production from goblet cells and causing a decrease in the mucociliary transport velocity.

CLINICS also publishes four Case Reports and one Technical Note. 